\title{
Patterning Multiple Aligned Self-Assembled Monolayers Using Light (Supporting Information)
}

Declan Ryan, ${ }^{\dagger}$ Babak Amir Parviz, ${ }^{\dagger}$ Vincent Linder ${ }^{\dagger}$ Vincent Semetey, ${ }^{\dagger}$ Samuel K. Sia, ${ }^{\dagger}$ Jing Su, ${ }^{\ddagger}$ Milan Mrksich, ${ }^{\ddagger}$ George M. Whitesides ${ }^{*}{ }^{\dagger}$

${ }^{\dagger}$ Department of Chemistry and Chemical Biology, Harvard University, 12 Oxford St., Cambridge, MA 02138.

$\$$ Department of Chemistry, University of Chicago, 5735 S. Ellis Avenue, Chicago, IL 60637.

*Corresponding author: gwhitesides@gmwgroup.harvard.edu 
Figure S1. SEM images of the photomask used for patterning multiple, aligned SAMs. (A) Wide-field view of the pattern of the photomask. (B) Magnified image of the region marked in (A) revealing the non-uniformity of the etch process. (C) A further magnification in the region marked in (B) reveals a gradient of chromium at the interface between the regions of metal and quartz. This gradient region is responsible for the blue fluorescent rim around the black regions in Figure 4B. 
Figure S1

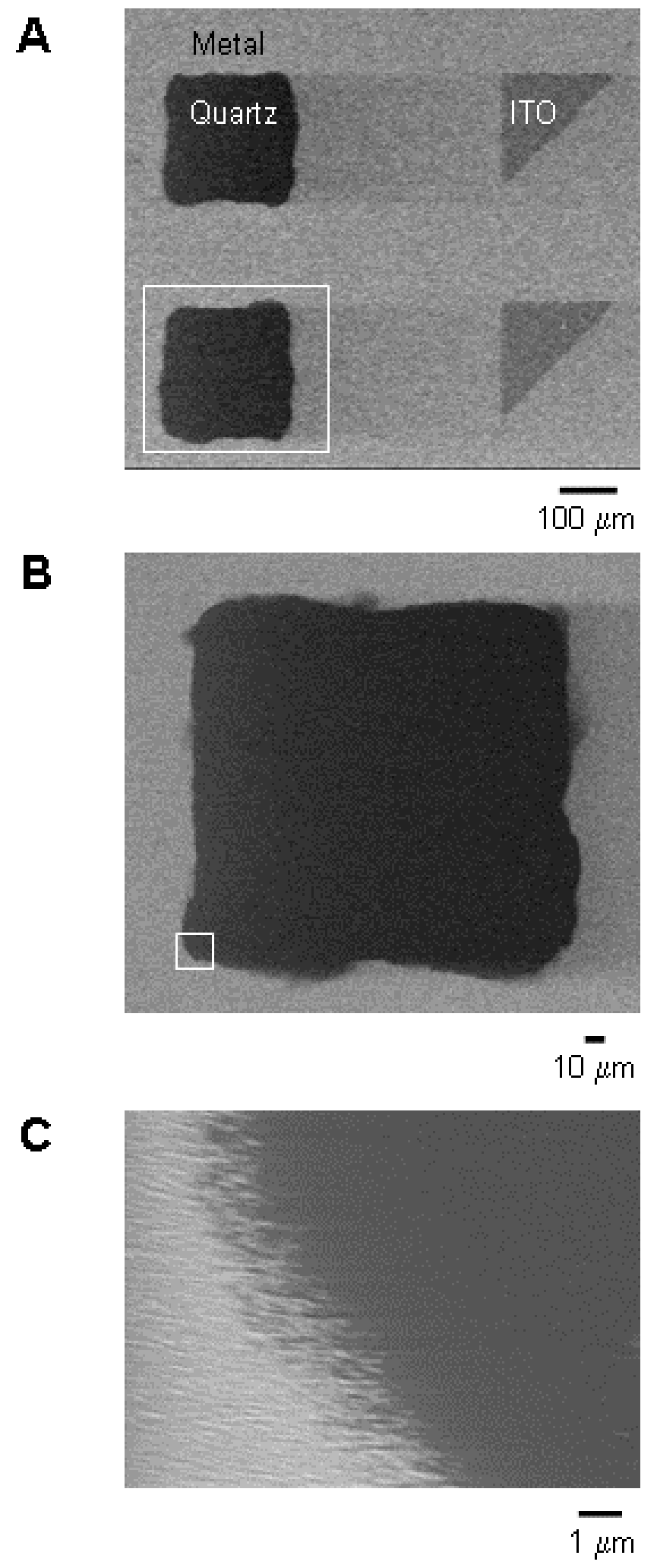

